Running head: Imitative and spatial compatibility

\title{
Time course analyses confirm independence of imitative and spatial compatibility
}

\author{
Caroline Catmur ${ }^{1, *}$ and Cecilia Heyes ${ }^{1,2}$ \\ ${ }^{1}$ ESRC Centre for Economic Learning and Social Evolution, University College London \\ ${ }^{2}$ Present address: All Souls College and Department of Experimental Psychology, University \\ of Oxford \\ *Corresponding author: c.catmur@ucl.ac.uk
}

Submitted to Journal of Experimental Psychology: Human Perception and Performance, $4^{\text {th }}$ August 2009. Revision submitted $7^{\text {th }}$ January 2010. Accepted $11^{\text {th }}$ January 2010.

\section{Author note}

Caroline Catmur, ESRC Centre for Economic Learning and Social Evolution, University College London, United Kingdom; and Cecilia Heyes, All Souls College and Department of Experimental Psychology, University of Oxford, United Kingdom, and ESRC Centre for Economic Learning and Social Evolution, University College London, United Kingdom.

This research was supported by funding from the European Community's Sixth Framework Programme under contract number NEST 012929, and the ESRC Centre for Economic Learning and Social Evolution. The experiments reported form part of the first author's doctoral thesis. Portions of these data were previously presented at the January 2009 meeting of the Experimental Psychology Society, London, United Kingdom. 
Correspondence concerning this article should be addressed to Caroline Catmur, ESRC

Centre for Economic Learning and Social Evolution, University College London, 26 Bedford Way, London WC1H 0AP, United Kingdom. E-mail: c.catmur@ucl.ac.uk 


\begin{abstract}
Imitative compatibility, or automatic imitation, has been used as a measure of imitative performance, and as a behavioural index of the functioning of the human mirror system (e.g. Brass, Bekkering, Wohlschlager, \& Prinz, 2000; Heyes, Bird, Johnson, \& Haggard, 2005; Kilner, Paulignan, \& Blakemore, 2003). However, the use of imitative compatibility as a measure of imitation has been criticised on the grounds that imitative compatibility has been confounded with simple spatial compatibility (Aicken, Wilson, Williams, \& Mon-Williams, 2007; Bertenthal, Longo, and Kosobud, 2006; Jansson, Wilson, Williams, \& Mon-Williams, 2007). Two experiments are reported in which, in contrast with previous studies, imitative compatibility was measured on both spatially compatible and spatially incompatible trials, and imitative compatibility was shown to be present regardless of spatial compatibility. Additional features of the experiments allowed measurement of the time courses of the imitative and spatial compatibility effects both within and across trials. It was found that imitative compatibility follows a different time course from spatial compatibility, providing further evidence for their independence and supporting the use of imitative compatibility as a measure of imitation.
\end{abstract}




\section{Keywords}

imitative compatibility, automatic imitation, spatial compatibility, mirror system, stimulusresponse compatibility 
Imitative compatibility is a particular type of stimulus-response compatibility effect in which both stimuli and responses consist of body movements. Imitative compatibility was first reported by Stürmer, Aschersleben, and Prinz (2000), who demonstrated that participants were faster to perform a hand opening movement while viewing a compatible (hand opening) movement, than when viewing an incompatible (hand closing) movement, and that this effect was reversed for the performance of hand closing movements. Similar effects have been reported by Brass and colleagues (Brass, Bekkering, \& Prinz, 2001; Brass, Bekkering, Wohlschlager, \& Prinz, 2000), Craighero, Bello, Fadiga, and Rizzolatti (2002), Edwards, Humphreys, and Castiello (2003), Vogt, Taylor, and Hopkins (2003), Heyes, Bird, Johnson, and Haggard (2005), Press and colleagues (Press, Bird, Flach, \& Heyes, 2005; Press, Gillmeister, \& Heyes, 2006; Press, Gillmeister, \& Heyes, 2007), Bertenthal et al. (2006), Bird, Leighton, Press, and Heyes (2007), Vainio, Tucker, and Ellis (2007), Gillmeister, Catmur, Liepelt, Brass, and Heyes (2008), Liepelt, von Cramon, and Brass (2008), and Longo and colleagues (Longo \& Bertenthal, 2009; Longo, Kosobud, \& Bertenthal, 2008).

Imitative compatibility has frequently been described as "automatic imitation" (e.g. Bertenthal et al., 2006; Bird et al., 2007; Longo et al., 2008; Longo \& Bertenthal, 2009; Heyes et al., 2005; Press et al., 2005; 2006; 2007). In this paper, we shall use the term imitative compatibility in order to avoid confusion with unconscious mimicry effects seen in social settings (e.g. Chartrand \& Bargh, 1999), which can also be described as automatic imitation. Terminology notwithstanding, the imitative compatibility effects described above are regarded as evidence of automatic (as opposed to intentional) imitation because the identity of the compatible or incompatible observed movement is always task-irrelevant. For example, in the study by Stürmer et al. (2000), the participant was instructed to open their own hand if the hand stimulus turned red, and to close their hand if it turned blue. At the same time, the stimulus hand performed an opening or closing movement. This movement, 
which could be compatible or incompatible with the movement performed by the participant, was irrelevant with respect to the participant's task. Brass et al. (2001) used a simple reaction time task where participants had to make the same movement on every trial within a block; the compatible or incompatible movement stimulus acted as an imperative stimulus or "go signal" for the participant to perform the prepared movement, telling participants when to move, but not what to do. In these experiments, participants were not required to process the identity of the observed movement - and indeed, in the case of incompatible movements, processing of movement identity was clearly counter-productive with respect to task performance - yet movement identity still had an effect on response times.

Further experiments have shown that observing another's movements interferes not only with response times but with performance accuracy. For example, Kilner, Paulignan and Blakemore (2003; see also Kilner, Hamilton, \& Blakemore, 2007) asked participants to move their arm in time with the observed movements of a human or robot arm. The stimulus arm moved compatibly (in the same plane) or incompatibly (at $90^{\circ}$ ) with the participants' movements. When observing the incompatible human movements, participants' movements showed significantly greater variance in the plane of the observed movements than in any of the other conditions.

The effects reported in the above studies arise as a result of the relationship between the observed stimulus movement and performed response movement. When observed and performed movements match, participants respond more quickly or accurately. It is the translation between observation and performance of the same movement which is the defining characteristic of imitation and thus these are regarded as imitation effects. 


\section{Uses of Imitative Compatibility}

The majority of the studies listed above have used imitative compatibility as an index of the functioning of the mirror system. Mirror neurons, discovered in the macaque monkey (di Pellegrino, Fadiga, Fogassi, Gallese, \& Rizzolatti, 1992; Gallese, Fadiga, Fogassi, \& Rizzolatti, 1996), fire both when the monkey executes a particular movement and when it passively observes the same movement. Converging evidence for a homologous mirror system in the human brain (Fadiga, Fogassi, Pavesi, \& Rizzolatti, 1995; Hari et al., 1998; Iacoboni et al., 1999; Iacoboni \& Mazziotta, 2007) has led to renewed interest in imitation as a behaviour which may rely on the key property of the mirror system: its ability to perform perceptual-motor translations between observed and executed movements (Catmur, Walsh, \& Heyes, 2009; Heiser, Iacoboni, Maeda, Marcus, \& Mazziotta, 2003).

Imitative compatibility has also been used as an index of the strength of the impulse or capacity to imitate in studies investigating the psychological processes mediating imitation. In this context, it is regarded as a relatively pure measure because, in comparison with intentional imitation tasks, imitative compatibility tasks minimise demands on motivational and working memory processes (Catmur et al., 2009).

Studies investigating psychological processes have used imitative compatibility to examine the role of ideomotor mechanisms in imitation (Brass et al., 2000; 2001; Stürmer et al., 2000); functional properties of imitation: e.g. whether it is modulated by the animacy, goaldirectedness and intentionality of observed actions (Liepelt et al., 2008; Longo et al., 2008; Longo \& Bertenthal, 2009; Press et al., 2005; 2006; Vainio et al., 2007); its developmental origins - for example, imitative compatibility studies suggest that imitation is the result of sensorimotor experience (Gillmeister et al., 2008; Heyes et al., 2005; Press et al., 2007); and 
its potential role in developmental disorders - Bird et al. (2007) demonstrated intact imitative compatibility in participants with autism spectrum disorder. Thus, imitative compatibility has been used to make a range of inferences about both the mirror system and the psychological processes mediating imitation.

\section{Spatial Compatibility Confounds Measures of Imitative Compatibility}

The use of imitative compatibility to measure the impulse to imitate rests on the assumption that imitative compatibility effects truly reflect processes of imitation. That is, that they provide a specific measure of the extent to which observation of a movement facilitates or interferes with the performance of the same or a different movement, where the similarity between movements depends on the configural spatial relationship between body parts. Such facilitation or interference is assumed to result from perceptual-motor translations between sensory and motor representations of movements: a necessary step during imitation, and one that may be performed by the mirror system (Catmur et al., 2009; Heiser et al., 2003).

One criticism that has been levelled at response time and interference studies of imitative compatibility is that these effects are often confounded with left/right or up/down spatial compatibility (Aicken, Wilson, Williams, \& Mon-Williams, 2007; Bertenthal et al., 2006; Jansson, Wilson, Williams, \& Mon-Williams, 2007), which would undermine their validity as a measure of imitation. For example, Brass et al. (2000) demonstrated imitative compatibility effects to task-irrelevant index and middle finger lifting movements when participants responded to symbolic cues by lifting their index or middle finger. This result could be explained by left/right spatial compatibility (Aicken et al., 2007; Bertenthal et al., 2006; Jansson et al., 2007) because the imitatively compatible pairing between the task-irrelevant stimulus and the response (e.g. observe index finger lift while performing index finger lift) 
was also spatially compatible (observe movement on left side of space while performing movement on left side of space), and the imitatively incompatible stimulus-response pairing was also spatially incompatible. The finding by Stürmer et al. (2000) of imitative compatibility effects to opening and closing hand movements could be explained, in a similar way to that of Brass et al. (2000), by up/down spatial compatibility (Jansson et al., 2007). In general, in any imitative compatibility experiment where stimulus movements are presented in the same spatial alignment as that in which the participants' response movements are made, spatial and imitative compatibility are confounded.

Attempts have been made to address this problem: Heyes et al. (2005) placed participants' response hands orthogonal to the direction of the observed stimuli; however, orthogonal spatial compatibility (Cho \& Proctor, 2004), where participants are faster to respond to a rightward stimulus with an upward response, and a leftward stimulus with a downward response, may still operate in this spatial configuration. Bertenthal et al. (2006, Experiment 1), in common with many other studies, found a large compatibility effect when spatial and imitative compatibility were confounded, which could be due to either the spatial or the imitative properties of the stimuli, or both. Therefore, in a separate experiment (Experiment 2), spatial and imitative compatibility were placed in opposition to each other, and only a spatial compatibility effect was seen. This might suggest that imitative compatibility does indeed result from spatial compatibility; but the spatial compatibility effect in this experiment was smaller than the combined compatibility effect in the first experiment, suggesting an influence of the conflicting imitative stimulus properties on the size of the spatial compatibility effect in Experiment 2. However, Experiments 1 and 2 were performed with different samples of participants and since there may be between-subjects differences in the sizes of the spatial compatibility effects, the conclusions that can be drawn from this study are limited. Brass et al. (2001, Experiment 3) used a within-subjects design: in two separate 
experimental sessions, they placed spatial and imitative compatibility in opposition to each other or in the same direction. When the data from both sessions were combined, Brass et al. (2001) found a greater imitative than spatial compatibility effect. However, participants may have learned to focus on either the spatial or the imitative properties of the movements in the session where these were in opposition, while they would not need to distinguish between these properties in the session where these properties were confounded. This difference between sessions might, therefore, have produced effects on responses which would not be seen if all trial types were presented in random order in the same experimental session.

Thus, as can be seen in Table 1, no previous study (neither those investigating imitative compatibility as an index of imitation, nor those critical of this approach) has addressed directly the potential confound between spatial and imitative compatibility, by assessing the influence of different levels of spatial and imitative compatibility in a randomised design within the same experimental session.

\begin{tabular}{|c|c|c|c|c|}
\hline \multirow[b]{4}{*}{ Experiments } & \multicolumn{4}{|c|}{ Trial Types } \\
\hline & \multicolumn{2}{|c|}{ Spatially Compatible } & \multicolumn{2}{|c|}{ Spatially Incompatible } \\
\hline & Imitatively & Imitatively & Imitatively & Imitatively \\
\hline & Compatible & Incompatible & Compatible & Incompatible \\
\hline Stürmer et al. (2000) & $\sqrt{ }$ & & & $\sqrt{ }$ \\
\hline Brass et al. (2000) & $\sqrt{ }$ & & & $\sqrt{ }$ \\
\hline Brass et al. (2001), Expts. 1 and 2 & $\sqrt{ }$ & & & $\sqrt{ }$ \\
\hline Brass et al. (2001), Expt. 3 "unflipped” session & $\sqrt{ }$ & & & $\sqrt{ }$ \\
\hline Brass et al. (2001), Expt. 3 "flipped" session & & $\sqrt{ }$ & $\sqrt{ }$ & \\
\hline Heyes et al. $(2005)^{1}$ & $\sqrt{ }$ & & & $\sqrt{ }$ \\
\hline Bertenthal et al. (2006), Expt. 1 & $\sqrt{ }$ & & & $\sqrt{ }$ \\
\hline Bertenthal et al. (2006), Expt. 2 & & $\sqrt{ }$ & $\sqrt{ }$ & \\
\hline Bertenthal et al. (2006), Expt. 3a & $\sqrt{ }$ & & $\sqrt{ }$ & \\
\hline
\end{tabular}


Bertenthal et al. (2006), Expt. 3b

Aicken et al. (2007), Expts. 1 and 2

Jansson et al (2007), Expts. 1 and $2^{1}$

Experiment 1

Experiment 2
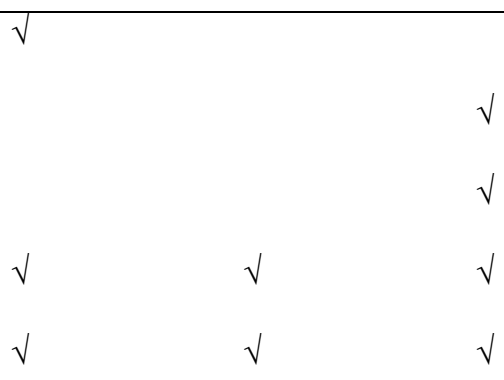

Table 1. Trial types used in previous experiments investigating imitative compatibility. It can be seen that no previous experiment has presented trials from both levels of spatial and imitative compatibility within the same experimental session. ${ }^{1}$ Heyes et al. (2005), and Jansson et al. (2007), Expt. 2, presented stimuli orthogonal to responses, but orthogonal spatial compatibility effects may still be seen in this configuration (Cho \& Proctor, 2004); therefore, these trials are classified as spatially compatible and incompatible.

\section{Dissociating Imitative Compatibility from Spatial Compatibility}

The experiments reported in the current paper tested the independence of imitative compatibility from spatial compatibility in two ways: using measures of mean response times, and time course analyses. We used a task in which each level of imitative compatibility was measured at each level of spatial compatibility, and all trial types were presented in randomised order within the same experimental session. The task was a choice reaction time task in which participants responded to the colour of a circle (discriminative stimulus) presented at fixation by making an outward (abduction) movement of either the index or the little finger of the right hand. Response times were measured using electromyography. Simultaneous with the onset of the discriminative stimulus, a taskirrelevant stimulus (a finger abduction movement) was presented on the screen. This movement could be of either the index or little finger, on either the right or the left hand. Thus, the task fulfilled the requirements for an imitative compatibility task: both the taskirrelevant stimuli and the responses consisted of configural body movements. It also fulfilled the requirements for a spatial compatibility task: both the task-irrelevant stimuli and the 
responses were aligned along the same (left-right) spatial dimension. (In the case of the responses and of the right hand stimuli, the index finger movement was on the left side of space and the little finger movement was on the right side of space; in the case of the left hand stimuli, the index finger movement was on the right side of space and the little finger movement was on the left side of space). The use of both left and right hand stimuli allowed manipulation of the spatial location of the stimulus independently of its imitative (finger identity) properties, resulting in all four of the trial types listed in Table 1. Table 2 illustrates how the task-irrelevant stimuli and the responses combined to make up these four trial types.

\begin{tabular}{|c|c|c|c|c|}
\hline & & Task-Irrelev & nt Stimulus & \\
\hline & Index finger & Little finger & Index finger & Little finger \\
\hline Response & Left side of space & Right side of space & Right side of space & Left side of space \\
\hline Index finger & imitatively & imitatively & imitatively & imitatively \\
\hline & compatible & incompatible & compatible & incompatible \\
\hline & spatially & spatially & spatially & spatially \\
\hline Left side of space & compatible & incompatible & incompatible & compatible \\
\hline Little finger & imitatively & imitatively & imitatively & imitatively \\
\hline & incompatible & compatible & incompatible & compatible \\
\hline & spatially & spatially & spatially & spatially \\
\hline Right side & incompatible & compatible & compatible & incompatible \\
\hline
\end{tabular}

Table 2. Imitative and spatial compatibility of trial types used in Experiments 1 and 2. Responses were always made with the right hand. The four trial types are indicated by different levels of shading. 
By including trial types that allowed measurement of each level of imitative compatibility (compatible, incompatible) at each level of spatial compatibility (compatible, incompatible), this design permitted the assessment of whether spatial compatibility and imitative compatibility are truly independent. For example, if previously reported imitative compatibility effects were the result of spatial compatibility, as suggested by Aicken et al. (2007) and Jansson et al. (2007), then when spatial compatibility is controlled in this fashion, an effect of spatial compatibility but no imitative compatibility effect will be observed. If, however, both spatial and imitative compatibility effects are observed when spatial compatibility is controlled, this would imply that spatial and imitative compatibility are independent of one another and thus that spatial and imitative compatibility are distinct phenomena.

Time Course Analyses of Spatial Compatibility and Imitative Compatibility

As well as a fully factorial design which allowed independent measurement of spatial and imitative compatibility, the two experiments reported in the current article had additional features to allow investigation of the time course of the spatial compatibility and imitative compatibility effects across the course of a trial. Experiment 1 contained sufficient trials to perform a quintile analysis (Ratcliff, 1979), in which, within each trial type, trials of differing response times could be compared. (Experiment 1 also included a discriminability variable: the task-relevant colour stimuli were strongly or weakly discriminable. This variable was intended to increase the range of response times (Hommel, 1994), but was not effective in doing so.) Experiment 2 used an offset factor that varied the timing of the discriminative stimulus with respect to the irrelevant movement stimulus. This factor was designed to manipulate the stage of processing reached by the irrelevant movement stimulus when responding was initiated. 
By performing a quintile analysis and manipulating the processing stage of the irrelevant movement stimulus, it was possible to assess the strengths of the spatial compatibility and imitative compatibility effects at different time points during the course of a trial. This provided another way of discriminating the two processes: if the spatial compatibility and imitative compatibility effects have different time courses, they are likely to represent distinct processes. Brass et al. (2001), using a quintile analysis, showed that both spatial compatibility and imitative compatibility effects grew larger as response times increased, but that the imitative compatibility effect increased more steeply with increasing response time. However, Jansson et al. (2007), in two separate experiments, failed to replicate this increase in imitative compatibility effects over time, from which they concluded that there was no evidence that imitative compatibility is distinct from spatial compatibility.

Experiment 1 therefore sought to establish the independence of imitative compatibility and spatial compatibility in two ways. The first was to assess whether imitative compatibility occurred when spatial compatibility was controlled. The second was to investigate, using a quintile analysis, whether the time course of imitative compatibility and spatial compatibility effects differed within the course of each trial. If, as suggested by Aicken et al. (2007) and Jansson et al. (2007), imitative compatibility is due to spatial compatibility, then a main effect of spatial compatibility but no effect of imitative compatibility should be observed. There should also be no difference in the time courses of the two effects, as measured using a quintile analysis. If, however, spatial compatibility and imitative compatibility are independent from one another, main effects of both spatial and imitative compatibility should be seen, and, consistent with Brass et al. (2001), the time courses of the two effects should differ. 


\section{Experiment 1}

Method

\section{Participants}

Sixteen right-handed volunteers (seven male), aged 19-35 years, took part. Participants were randomly allocated to receive either high or low discriminative stimulus discriminability (see Stimuli). Two additional participants were removed from the sample prior to data analysis, due to insufficient data (subject error or poor electrode signal on more than $20 \%$ of trials). For both Experiments 1 and 2, participants were recruited using the University College London (UCL) Psychology Department subject pool, and paid for their participation; the experiments were approved by the UCL Ethics Committee, and all participants gave written informed consent before participating.

\section{Stimuli}

The stimuli were video files made up of two still images of a female left or right hand. The hand was displayed initially in a neutral (resting) position, and subsequently in the (taskirrelevant) final movement position, which consisted of an abduction movement of either the index or little finger (see Table 2 and Figure 1A). The movement was made in the horizontal plane, i.e. the plane of the hand and fingers, and was shown as if viewed from above. Videos (720 by 576 pixels) were constructed using Adobe Premiere (Adobe Systems Incorporated, San Jose, California, USA). The replacement of the neutral stimulus by the final movement position produced apparent motion, which has been shown to give robust imitative compatibility effects (Press et al., 2005; Stürmer et al., 2000) while allowing greater experimental control of movement stimulus onset than gradual progression of the movement. The hand was presented on a black background and subtended a visual angle of $14.9^{\circ}$ 
vertically and between $7.7^{\circ}$ (neutral) and $9.2^{\circ}$ (little finger movement) horizontally, when viewed at a distance of $57 \mathrm{~cm}$. The finger movements subtended an angle of $17^{\circ}$ (index) and $29^{\circ}$ (little) from the neutral position. The left hand videos were created by reflecting the right hand images in the y-axis and were identical to the right hand videos in all other respects.

The onset of the discriminative stimulus, telling the participant whether to respond with their index or little finger, was simultaneous with the onset of the (task-irrelevant) movement stimulus. The discriminative stimulus consisted of a solid, coloured circle, occupying $\sim 1^{\circ}$ visual angle. Prior to the onset of the coloured circle, its location was indicated by the presence of the outline of a white circle, also $\sim 1^{\circ}$ visual angle, which acted as a fixation point. This location was at a point equidistant between the tips of the index and little fingers in the neutral position, thus ensuring that spatial attention was equal between the two fingers, and giving no information about the subsequent movement. In order to make the discrimination task relatively difficult, the two colours of the discriminative stimulus, indicating the two responses, were chosen to be similar. The mean colour of the hand stimulus was calculated by finding the mean intensity of the red, green and blue components of every coloured pixel in the hand image. For half of the participants, the red component of this colour was incremented by 32 (on a scale of 1:256) to produce an "orange" colour, while the blue component was incremented by the same amount to produce a "purple" colour. For the other eight participants, these components were incremented by 16 on the same scale, in order to create two levels of discriminability (high: incremented by $32 / 256$; low: incremented by $16 / 356)$ between participants. 
A.

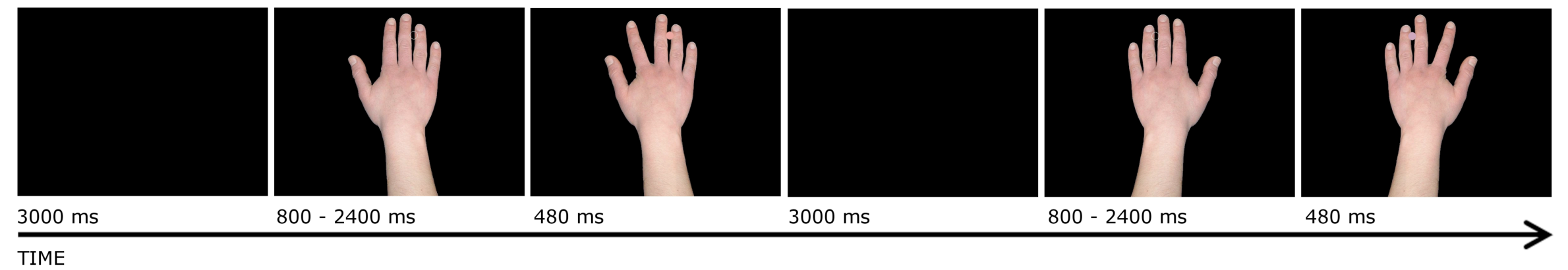

B.

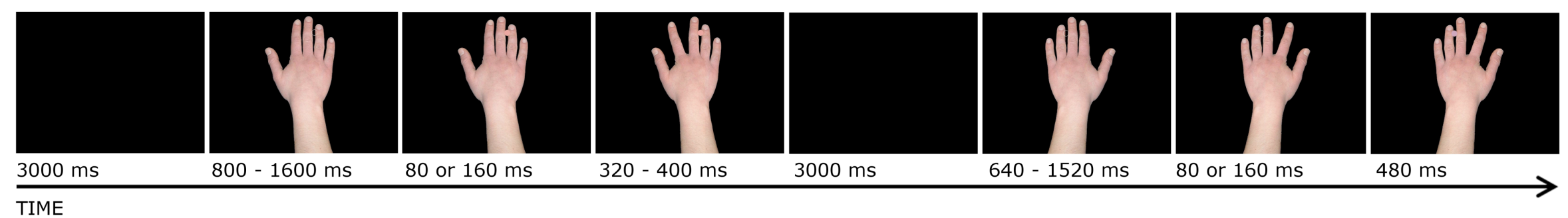

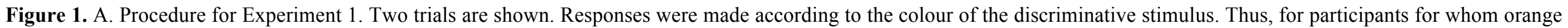

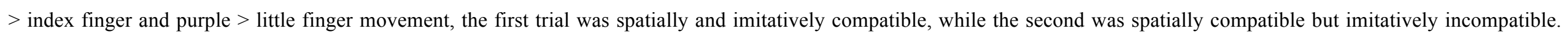

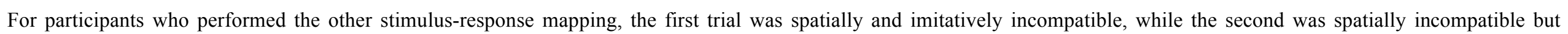

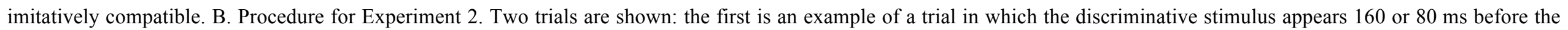




\section{Apparatus}

Stimuli were presented on a Dell Latitude D800 laptop (Dell Incorporated, Round Rock, Texas, USA). Time of onset of the final movement position and (simultaneously) the discriminative stimulus was identified by a signal sent via the parallel port to the data acquisition computer. This triggered data acquisition and allowed response time (RT) to be calculated with respect to stimulus onset time.

\section{Procedure}

Participants were seated approximately $60 \mathrm{~cm}$ from the stimulus presentation screen. All responses were made with the right hand. Their right arm was supported from the elbow to the palm by an armrest, placed such that their right hand was in the same orientation as the hand on the screen (with the wrist closest to the participant and the fingertips furthest away). This was to ensure spatial compatibility or incompatibility between the observed and performed movements on the relevant trials. Participants were instructed to fixate on the white circle which was presented on the hand in the neutral position on every trial. They were informed that the circle would change to a coloured circle, and that this indicated that they should make an abduction movement of either the index or the little finger. The stimulusresponse mappings (orange $>$ index finger, purple $>$ little finger, or vice versa), and discriminability of circle colour (high or low) were fully counterbalanced between participants. Participants were encouraged to perform the movements as fast as possible without sacrificing accuracy.

Each trial began with the video of the neutral hand position, which was presented for one of three stimulus onset asynchronies (SOAs: 800, 1600, or $2400 \mathrm{~ms}$ ). This was followed by the final movement position and discriminative stimulus, which remained on the screen for $480 \mathrm{~ms}$. A blank screen was then presented for $3000 \mathrm{~ms}$ before the next trial began (see 
Figure 1A). The different trials were made up of a factorial combination of stimulus movement (index or little), stimulus movement location (left or right side of the screen), and response movement (index or little, instructed by the colour of the discriminative stimulus). A total of 288 trials were presented in a random order in four blocks of 72 trials. Each of the main four trial types (as listed in Tables 1 and 2) was presented 18 times in every block, three times for each combination of response movement and SOA. Before the start of the experiment, participants were given the chance to practice making the two finger movements, during which time they received visual feedback of their electromyogram (EMG) signal. They then received 24 practice trials in a random order to familiarise them with the format of the experiment, with each of the four trial types presented once for each combination of response movement and SOA. No visual EMG feedback was given during either practice or experimental trials.

\section{Data acquisition and analysis}

The EMG was recorded from the first dorsal interosseous and abductor digiti minimi muscles of the right hand, which control abduction of the index and little fingers, respectively. Pairs of disposable $\mathrm{Ag}-\mathrm{AgCl}$ electrodes (Unomedical a/s, Birkerød, Denmark) were attached to these muscles in a belly-tendon montage, with a third (common input) electrode placed on the wrist. Signals were amplified at a gain of 1,000 x using a 1902 amplifier (Cambridge Electronic Design, Cambridge, UK), band-pass filtered between 20 and 2,000 Hz and mainshum filtered at $50 \mathrm{~Hz}$. A second laptop (Dell Latitude C400) used a data acquisition card (DAQCard-PCI-6024E, National Instruments Corporation, Austin, Texas) and a Matlab script (The Mathworks, Natick, Massachusetts, USA) to sample these signals at $3 \mathrm{kHz}$ and record them for later analysis. 
For every trial, RT was calculated by moving a $20 \mathrm{~ms}$ window across the EMG data in $1 \mathrm{~ms}$ increments. The standard deviation of the EMG signal within this window was calculated and compared to the standard deviation of the signal in the $100 \mathrm{~ms}$ before stimulus onset (the baseline period). Once the standard deviation of the data in the $20 \mathrm{~ms}$ window was over 2.75 times that of the baseline period for three successive $20 \mathrm{~ms}$ windows, the end of the first window was taken as the end of the RT period. Whether this time point accurately reflected the onset of the EMG response was verified by eye for every trial performed by every participant.

Results and Discussion

Trials on which participants made an error or took more than $1000 \mathrm{~ms}$ to respond $(2.5 \%)$ were excluded from analysis. Trials on which the analysis program failed accurately to detect the onset of the EMG response $(6.1 \%)$ were also excluded. Mean RT was calculated for each of the four trial types, collapsed across the two different response movements. Figure 2 shows the RT and error data.

A

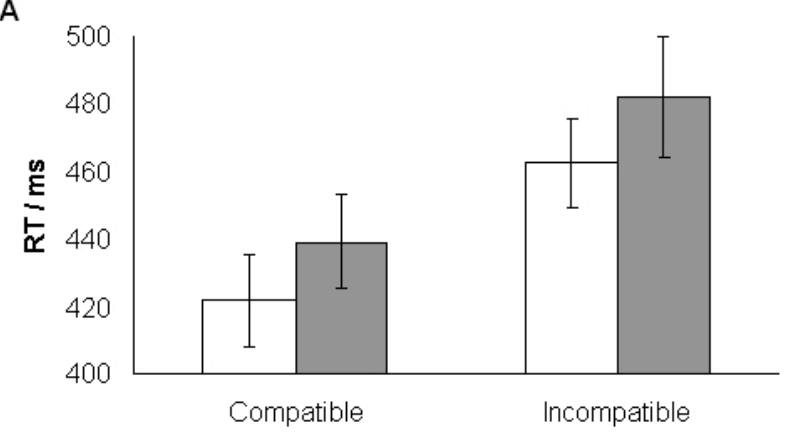

Spatial compatibility

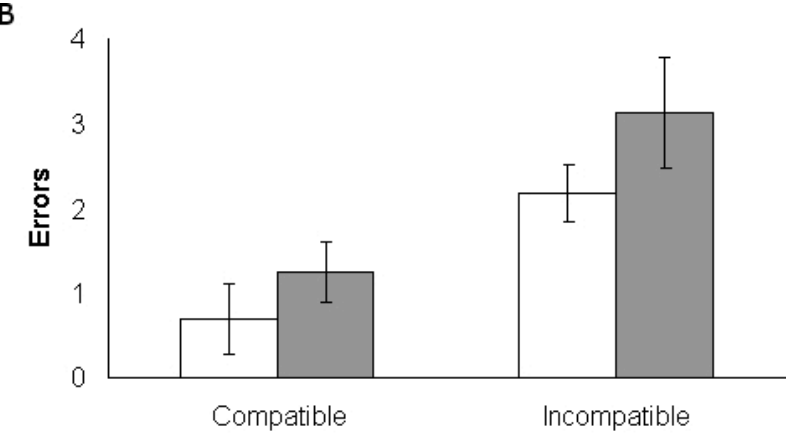

Spatial compatibility

$\square$ Imitatively compatible $\square$ Imitatively incompatible

Figure 2. Mean \pm SEM of RT (A) and errors (B) for Experiment 1. Data are shown for the four trial types, i.e. each level of imitative compatibility at each level of spatial compatibility. 
A repeated measures analysis of variance (ANOVA) was performed on the RT data. The within-subjects factors were spatial compatibility (compatible, incompatible) and imitative compatibility (compatible, incompatible). The between-subjects factor was the discriminability of the discriminative stimulus (high, low). Here and subsequently, all significant main effects and interactions are reported ( $\alpha=0.05$ unless otherwise stated). There was a significant main effect of spatial compatibility: participants responded faster on trials where the irrelevant movement stimulus was spatially compatible with the response (mean \pm standard error of the mean (SEM): $431 \pm 14 \mathrm{~ms}$ ) compared to where it was spatially incompatible $\left(472 \pm 15 \mathrm{~ms} ; F_{1,14}=63.8, p<0.001\right)$. There was also a significant main effect of imitative compatibility: participants responded faster on trials where the irrelevant movement stimulus was performed with the same finger as the response (442 $\pm 13 \mathrm{~ms})$ than on trials where it was performed with the other finger $\left(461 \pm 16 \mathrm{~ms} ; F_{1,14}=13.2, p=0.003\right)$. The two effects did not interact. There was no main effect of discriminability, and no interactions involving this factor.

A repeated measures ANOVA with the same factors was performed on the error data. There was a significant main effect of spatial compatibility: participants made more errors on spatially incompatible $(2.7 \pm 0.5)$ than on spatially compatible trials $\left(1.0 \pm 0.4 ; F_{1,14}=29.1\right.$, $p<0.001)$. The direction of this effect is such as to rule out a speed/accuracy trade-off that might otherwise account for the RT data.

The results of the RT analysis indicate that, contrary to the suggestions of Aicken et al. (2007) and Jansson et al. (2007), imitative compatibility is independent of spatial compatibility. If imitative compatibility were due solely to simple spatial compatibility, no main effect of imitative compatibility would have been observed when imitative 
compatibility was measured on both spatially compatible and spatially incompatible trials. Instead, main effects of both spatial and imitative compatibility were found.

In order to investigate the time course of the spatial compatibility and imitative compatibility effects within trials, a quintile analysis was performed (after Ratcliff, 1979). The distribution of each participant's RTs over the entire experiment, within each of the four trial types, was ordered by response speed and divided into five "bins" ( 1 = fastest to $5=$ slowest $)$ with an equal number of trials in each bin. The spatial compatibility effect (RT on spatially incompatible - RT on spatially compatible trials) and imitative compatibility effect (RT on imitatively incompatible - RT on imitatively compatible trials) were then calculated for each of the five quintiles. This allowed measurement of the size of the compatibility effects across the range of fast to slow RTs, providing an insight into the relative strength of each effect over time within a trial. ANOVA with within-subjects factors of quintile $(1-5)$ and compatibility modality (spatial, imitative) revealed a main effect of modality: the spatial compatibility effect was larger than the imitative compatibility effect (42 $\pm 6 \mathrm{~ms}$ compared to $\left.18 \pm 6 \mathrm{~ms} ; F_{1,15}=22.6, p<0.001\right)$. There was, importantly, an interaction between response speed and modality $\left(F_{4,60}=3.8, p=0.008\right)$ : simple effects analysis showed that the spatial compatibility effect was not affected significantly by increasing $\mathrm{RT}\left(F_{4,60}=1.2, p=0.321\right)$, while the imitative compatibility effect became larger as RT increased $\left(F_{4,60}=2.9, p=0.028\right)$ (see Figure 3). 


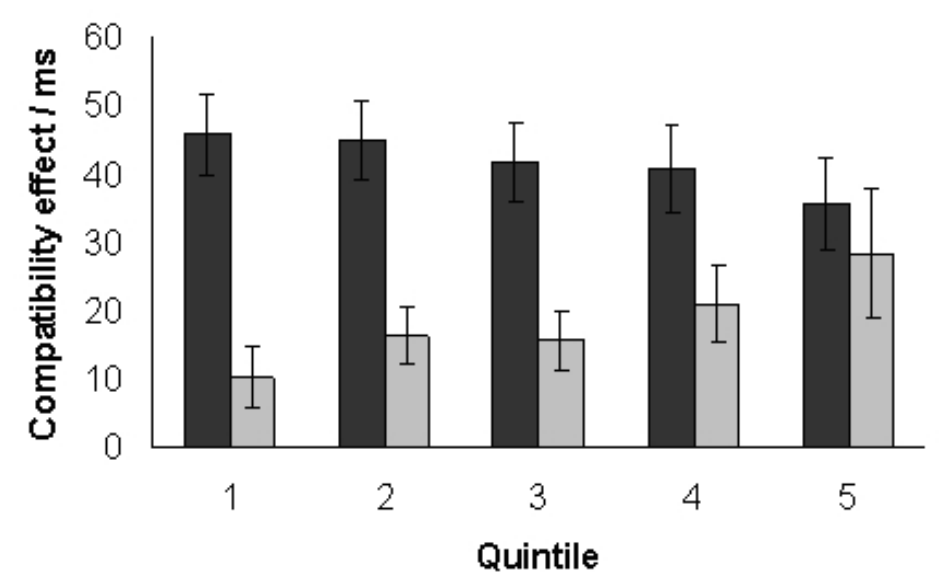

- Spatial compatibility effect $\square$ Imitative compatibility effect

Figure 3. Mean \pm SEM of size of spatial compatibility and imitative compatibility effects across the five quintiles $(1=$ fastest RTs, $5=$ slowest RTs) in Experiment 1.

The quintile analysis yielded three interesting results. First, the spatial compatibility effect was greater than the imitative compatibility effect. This is in contrast with the results of Brass et al. (2001, Experiment 3) who found a greater imitative compatibility effect than spatial compatibility effect. One possible reason for this difference is that the experiment by Brass et al. (2001) manipulated up/down, rather than left/right, spatial compatibility; it is possible that certain types of spatial representations are more effective than others in eliciting compatibility effects (Nicoletti \& Umiltà, 1984). This explanation is in line with the findings of Bertenthal et al. (2006, Experiments 3a and 3b), in which left/right stimulus arrangements also produced larger spatial compatibility effects than imitative compatibility effects. In addition, the current stimuli displayed a greater degree of spatial eccentricity than those of Brass et al. (2001), which could also explain the stronger spatial compatibility effect.

The second result of the quintile analysis was that the imitative compatibility effect increased as RT increased. Thirdly and most importantly, increases in RT affected the sizes of the spatial compatibility and imitative compatibility effects differentially: in contrast with the imitative compatibility effect, the spatial compatibility effect did not increase with increasing 
RT. These two results are consistent with the findings of Brass et al. (2001) but at odds with Jansson et al. (2007) who did not find an effect of RT on the size of the imitative compatibility effect.

Experiment 1 therefore confirmed that spatial compatibility and imitative compatibility are independent of one another and that these effects follow distinct time courses within each trial.

\section{Experiment 2}

Experiment 2 aimed to replicate and extend the findings of Experiment 1 by using, within the same experimental task, a convergent method to investigate the time courses of the spatial compatibility and imitative compatibility effects. Experiment 2 used the same stimuli, task and levels of spatial and imitative compatibility as Experiment 1, with the exception that a timing manipulation was included: offset between the discriminative stimulus and irrelevant movement stimulus. By manipulating response time with respect to the irrelevant movement stimulus, it was possible to investigate the build-up and/or decay of the spatial compatibility and imitative compatibility effects over time within a trial. Hommel (1993; 1994), in a spatial compatibility task, presented the discriminative stimulus $196 \mathrm{~ms}$ after the irrelevant spatial stimulus. This manipulation delayed the response time with respect to the processing of the irrelevant spatial stimulus, which resulted in a reduced spatial compatibility effect. This result suggests that the stimulus-related activation which generates a left-right spatial compatibility effect decays within this period of time. In Experiment 2, a similar manipulation was used: time of presentation of the discriminative stimulus was varied with respect to the onset of the irrelevant movement stimulus. 
The time difference between the onsets of the discriminative and irrelevant movement stimuli was manipulated across five levels (offsets), in order to obtain as clear a picture as possible of any differences between the time courses of the two effects. Hommel's $(1993 ; 1994)$ data suggested that a delay of $196 \mathrm{~ms}$ between the onset of the irrelevant movement stimulus and the discriminative stimulus was sufficient for the decay of the spatial compatibility effect. In order to investigate the intermediate stages of this decay, levels of offset giving delays of $80 \mathrm{~ms}$ and $160 \mathrm{~ms}$ were chosen whereby the discriminative stimulus was presented after the irrelevant movement stimulus. Additionally, one simultaneous level of offset (identical to Experiment 1), and two levels where the discriminative stimulus was presented $80 \mathrm{~ms}$ or $160 \mathrm{~ms}$ before the irrelevant movement, were used. These "before" levels of offset were used in order to compare the initial stages, i.e. the build-up, of the time courses of the two effects, since Experiment 1 showed that both spatial compatibility and imitative compatibility effects were already present when the discriminative stimulus was presented simultaneously with the irrelevant movement stimulus.

Experiment 1 found that the imitative compatibility effect, unlike the spatial compatibility effect, increased with increasing RT. It was therefore predicted that the later ("after") levels of offset would show a greater imitative compatibility effect than the simultaneous or "before" levels, while the spatial compatibility effect might build up earlier and thus already be present at the "before" levels of offset.

Method

\section{Participants}

Eight right-handed volunteers (three male), aged 20-27 years, participated. 


\section{Stimuli}

The stimuli were identical to those used in Experiment 1 with the exception that the coloured circles did not vary in discriminability across participants (the higher discriminability stimuli from Experiment 1 were used).

\section{Apparatus}

The apparatus was identical to that used in Experiment 1 with the exception that data acquisition was triggered at the time of onset of the discriminative stimulus, irrespective of when the irrelevant movement stimulus was presented.

\section{Procedure}

The procedure was the same as Experiment 1 with the following exceptions. The video of the still hand was presented for one of two SOAs ( 800 or $1600 \mathrm{~ms})$, after which time the discriminative stimulus was presented. The discriminative stimulus was presented at one of five offsets with respect to the irrelevant movement stimulus (160 ms before, $80 \mathrm{~ms}$ before, simultaneous, $80 \mathrm{~ms}$ after, $160 \mathrm{~ms}$ after). Thus, the irrelevant movement stimulus could appear shortly after, at the same time as, or shortly before the discriminative stimulus (see Figure 1B).

A total of 560 trials were presented in a random order in 14 blocks of 40 trials. Trials were counterbalanced across sets of two blocks, such that each combination of trial type, response movement, and offset was presented twice in every two blocks, once for each SOA. Twelve randomly selected practice trials were given before the start of the experiment. 


\section{Results and Discussion}

Trials on which participants made an error or took more than $1000 \mathrm{~ms}$ to respond $(2.0 \%)$ were excluded from analysis. Trials on which the analysis program failed accurately to detect the onset of the EMG response $(0.5 \%)$ were also excluded. Mean RT was calculated for each of the combinations of trial type and offset (see Table 3) and the values of the spatial compatibility and imitative compatibility effects were then calculated for each offset (see Figure 4).

\begin{tabular}{|c|c|c|c|c|c|c|c|c|c|}
\hline \multirow[b]{5}{*}{ Offset } & \multirow{5}{*}{$\begin{array}{c}\text { Overall } \\
\text { RT }\end{array}$} & \multicolumn{8}{|c|}{ Trial Types } \\
\hline & & \multicolumn{4}{|c|}{ Spatially Compatible } & \multicolumn{4}{|c|}{ Spatially Incompatible } \\
\hline & & \multicolumn{2}{|c|}{ Imitatively } & \multicolumn{2}{|c|}{ Imitatively } & \multicolumn{2}{|c|}{ Imitatively } & \multicolumn{2}{|c|}{ Imitatively } \\
\hline & & \multicolumn{2}{|c|}{ Compatible } & \multicolumn{2}{|c|}{ Incompatible } & \multicolumn{2}{|c|}{ Compatible } & \multicolumn{2}{|c|}{ Incompatible } \\
\hline & & RT & Errors & RT & Errors & RT & Errors & RT & Errors \\
\hline $160 \mathrm{~ms}$ before & $437 \pm 14$ & $420 \pm 14$ & $0.1 \pm 0.1$ & $431 \pm 15$ & $0.3 \pm 0.2$ & $450 \pm 11$ & $0.4 \pm 0.3$ & $449 \pm 18$ & $1.0 \pm 0.3$ \\
\hline $80 \mathrm{~ms}$ before & $442 \pm 16$ & $431 \pm 16$ & $0.3 \pm 0.2$ & $417 \pm 15$ & $0.4 \pm 0.2$ & $458 \pm 16$ & $0.4 \pm 0.2$ & $462 \pm 17$ & $0.4 \pm 0.2$ \\
\hline Simultaneous & $432 \pm 17$ & $406 \pm 16$ & $0.3 \pm 0.3$ & $417 \pm 15$ & $0.9 \pm 0.2$ & $441 \pm 18$ & $0.3 \pm 0.2$ & $464 \pm 18$ & $1.5 \pm 0.5$ \\
\hline $80 \mathrm{~ms}$ after & $421 \pm 15$ & $383 \pm 17$ & $0.1 \pm 0.1$ & $420 \pm 12$ & $0.3 \pm 0.2$ & $425 \pm 16$ & $0.5 \pm 0.3$ & $457 \pm 15$ & $1.6 \pm 0.6$ \\
\hline $160 \mathrm{~ms}$ after & $412 \pm 15$ & $387 \pm 17$ & $0.1 \pm 0.1$ & $405 \pm 14$ & $0.5 \pm 0.3$ & $416 \pm 18$ & $0.4 \pm 0.2$ & $441 \pm 13$ & $1.5 \pm 1.0$ \\
\hline
\end{tabular}

Table 3. Mean \pm SEM of RT (ms) and number of errors in Experiment 2. RT and error data are shown for each of the four trial types at each of the five levels of offset, and overall RT for each level of offset. 


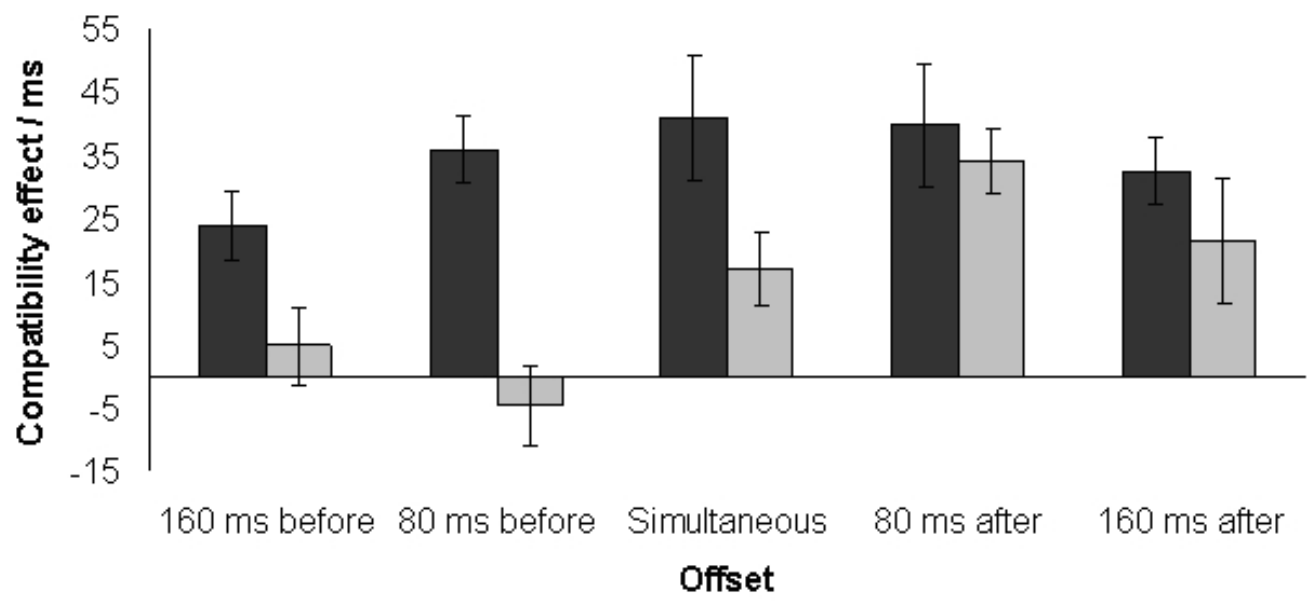

Spatial compatibility effect $\square$ Imitative compatibility effect

Figure 4. Mean \pm SEM of size of spatial compatibility and imitative compatibility effects for the five levels of offset in Experiment 2.

ANOVA with within-subjects factors of offset between discriminative and irrelevant stimuli (discriminative stimulus $160 \mathrm{~ms}$ before irrelevant movement, $80 \mathrm{~ms}$ before, simultaneous, $80 \mathrm{~ms}$ after, $160 \mathrm{~ms}$ after), spatial compatibility (compatible, incompatible), and imitative compatibility (compatible, incompatible), was performed on the RT data.

Replicating the results of Experiment 1, there was a significant main effect of spatial compatibility ( $412 \pm 15 \mathrm{~ms}$ compared to $\left.446 \pm 16 \mathrm{~ms} ; F_{1,7}=44.6, p<0.001\right)$ and of imitative compatibility ( $422 \pm 16 \mathrm{~ms}$ compared to $\left.436 \pm 15 \mathrm{~ms} ; F_{1,7}=24.6, p=0.002\right)$, and no interaction between these factors. There was also a significant main effect of offset: participants responded faster, the later the discriminative stimulus appeared with respect to the irrelevant movement $\left(F_{4,28}=11.4, p<0.001\right)$. There was a significant interaction between imitative compatibility and offset $\left(F_{4,28}=4.7, p=0.005\right)$. This interaction is illustrated in Figure 4: the imitative compatibility effect was not evident until the simultaneous and later levels of offset. In contrast, the spatial compatibility effect was relatively constant across all levels of offset. 
In order to investigate the interaction between imitative compatibility and offset, post-hoc $t$ tests (Bonferroni corrected: $\alpha=0.005$ ) were used to establish which levels of offset produced significantly different sizes of the imitative compatibility effect. There was one significant difference between offsets, which indicated that the interaction between imitative compatibility and offset was primarily driven by the difference in size of the imitative compatibility effects at offset levels $80 \mathrm{~ms}$ before and $80 \mathrm{~ms}$ after $\left(t_{7}=4.4, p=0.003\right)$, confirming a later build-up of the imitative compatibility effect.

The error data were subjected to ANOVA with the same within-subjects factors of offset between discriminative and irrelevant stimuli (discriminative stimulus $160 \mathrm{~ms}$ before irrelevant movement, $80 \mathrm{~ms}$ before, simultaneous, $80 \mathrm{~ms}$ after, $160 \mathrm{~ms}$ after), spatial compatibility (compatible, incompatible), and imitative compatibility (compatible, incompatible). There were significant main effects of spatial compatibility: participants made more errors on spatially incompatible trials than on spatially compatible trials $(0.8 \pm 0.4$ compared to $\left.0.3 \pm 0.2 ; F_{1,7}=6.7, p=0.036\right)$ and of imitative compatibility: participants made more errors on imitatively incompatible trials than on imitatively compatible trials $(0.8 \pm 0.3$ compared to $\left.0.3 \pm 0.2 ; F_{1,7}=7.0, p=0.033\right)$. Both of these effects were in such a direction as to rule out any speed/accuracy trade-off.

The main RT effects of spatial and imitative compatibility replicated the findings of Experiment 1 and confirmed the independence of spatial compatibility and imitative compatibility. The main effect of offset on RT may indicate that in the conditions where the onset of the irrelevant movement preceded the onset of the discriminative stimulus, participants were at a greater state of readiness to respond than in the other conditions, and hence were faster. 
Imitative compatibility showed an interaction with offset, indicating that the size of the imitative compatibility effect changed over the five levels of offset, as would be expected if the imitative compatibility effect built up over time. The post-hoc analyses indicated that the interaction between offset and imitative compatibility was driven by the difference between the $80 \mathrm{~ms}$ before and $80 \mathrm{~ms}$ after levels of offset. This result shows that the imitative compatibility effect built up later within a trial than the spatial compatibility effect, which was consistent across levels of offset. This later appearance of the imitative compatibility effect than of the spatial compatibility effect is consistent with the results of the quintile analysis presented in Experiment 1.

General Discussion

The experiments reported in the current article showed that, contrary to the suggestions of Aicken et al. (2007) and Jansson et al. (2007), imitative compatibility is independent of simple spatial compatibility. This result validates the use of imitative compatibility to assess imitative ability and performance. It also suggests that in previous studies in which spatial and imitative compatibility were confounded, the observed compatibility effect may have resulted from the combination of spatial compatibility and imitative compatibility.

Experiments 1 and 2 also indicated that processes of spatial compatibility and imitative compatibility display differing time courses within each trial, as reported by Brass et al. (2001), but contrary to Jansson et al. (2007). Spatial compatibility effects were present from the early stages of a trial, while imitative compatibility effects arose later in a trial (Experiment 2) and appeared to increase in size for longer than spatial compatibility effects (Experiment 1). 


\section{Psychological Mechanisms of Stimulus-Response Compatibility}

What do the results of the current experiments imply about the psychological mechanisms underlying spatial and imitative stimulus-response compatibility? One explanation could be that the two types of compatibility are the result of two entirely distinct mechanisms. An alternative explanation would be that both spatial compatibility and imitative compatibility arise from the same mechanism, but that the inputs to this mechanism differ in the case of the two different compatibility effects.

An influential model of stimulus-response compatibility (Kornblum, Hasbroucq, and Osman, 1990) suggests that it arises in the following manner: a stimulus produces intentional (controlled) activation of the task-relevant response as a result of task instructions, but also automatic (direct) activation of the irrelevant response. Conflict between these two response codes slows response preparation in the condition where the task-relevant and irrelevant responses are incompatible, leading to slower responses on incompatible than on compatible trials. However, the issue of why the irrelevant stimulus attributes activate the irrelevant response (for example, in spatial stimulus-response compatibility, why a stimulus on the left activates the left response) is not addressed by this model.

Tagliabue, Zorzi, Umiltà, and Bassignani (2000) demonstrated that spatial stimulus-response compatibility effects could be eliminated (in adults) or even reversed (in children) following a short period of training on the incompatible stimulus-response pairing (e.g. a stimulus on the right was responded to with a left button press). This result, and in particular the difference between the effects of incompatible training on children and on adults, suggests that in the case of spatial compatibility, the automatic activation of the response associated 
with the irrelevant stimulus may be the result of associative learning of spatial stimulusresponse contingencies during development.

Heyes et al. (2005) demonstrated a similar effect of training with imitatively incompatible stimulus-response pairs (e.g. a hand opening stimulus was responded to by closing the hand) on imitative compatibility: a short period of incompatible training abolished the imitative compatibility effect. This result is consistent with the associative sequence learning (ASL) theory of imitation (Heyes \& Ray, 2000), which proposes that imitation arises as a result of associative learning of stimulus-response contingencies between the observation and performance of movements.

\section{A Possible Common Psychological Mechanism}

The results presented in this paper, demonstrating imitative compatibility when spatial compatibility is controlled, indicate that imitative compatibility is not due to simple spatial compatibility. However, given the evidence that both spatial and imitative compatibility effects can be abolished by incompatible training, it is possible that the same kind of mechanism gives rise to both simple spatial compatibility and imitative compatibility - a mechanism consisting of links between sensory and motor representations established through domain-general processes of associative learning (Brass et al., 2000; Hommel, Musseler, Aschersleben, \& Prinz, 2001; Stürmer et al., 2000).

In the case of spatial compatibility, general theories of associative learning predict stronger associations between spatially compatible stimuli and responses than between spatially incompatible stimuli and responses because, during development, compatible stimulusresponse pairings are more common than incompatible pairings. For example, observation of 
an object at a particular spatial location is highly correlated with reaching movements towards that location, rather than towards other locations. In addition, observation of one's own performance of a movement in a particular spatial location will result in a high contingency between the activation of the sensory and motor representations of that spatial location. Orienting responses to lateralised stimuli will also produce spatially compatible stimulus-response pairings (Tagliabue et al., 2000).

The ASL theory suggests that in the case of imitative compatibility, links between sensory and motor representations of a movement arise from sensorimotor experience, during which the sensory and motor representations of the same movement are activated in a contingent manner. Such experience includes observing the outcome of your own actions, or being imitated by someone else (especially during infancy; Ray \& Heyes, in press). In both cases, motor and sensory representations of the same movement are contingently active: that is, the probability of observing the same movement as that which you are performing is higher than the probability of observing any other movement. The importance of contingency, rather than contiguity, in associative learning theory, is that it prevents associations being formed haphazardly between any two representations which happen to be active at the same time: there must be a contingent relationship between the activation of the two representations.

It may be, therefore, that in the case of both imitative and spatial compatibility, the psychological mechanism underlying the compatibility effects is the same: general processes of associative learning produce links between sensory representations (of a movement or of a spatial location) and motor representations (of the same movement or of a response in the same spatial location). The subsequent presentation of a stimulus with movement or spatial properties activates the associated response, resulting in imitative or spatial compatibility effects as a result of general processes of stimulus-response compatibility. (A similar general 
psychological mechanism which explains both types of compatibility is proposed by the Theory of Event Coding; Hommel et al., 2001). Note, however, that since the same psychological mechanism could be implemented in different neural locations, this does not necessarily mean that the two types of compatibility effect should occur in the same cortical area.

If compatibility effects are indeed the result of a common mechanism, this would suggest that the two different compatibility effects demonstrated in the current experiments arise from differing inputs to this general-purpose mechanism: the side of space in the case of spatial compatibility, versus a configuration of body parts moving in space in the case of imitative compatibility. These different inputs are likely to be processed at different rates, with more complex body part configurations taking longer to process than more simple information about the side of space of the irrelevant stimulus. It is possible, therefore, that this differential processing speed could explain why the time courses of the spatial compatibility and imitative compatibility effects differ within the course of each trial.

Evidence for Differing Psychological Mechanisms?

An alternative view has been put forward by Bertenthal et al. (2006), who suggested that imitative compatibility and spatial compatibility are mediated by differing, but as yet unspecified, mechanisms. Bertenthal et al. (2006, Experiments $3 \mathrm{a}$ and $3 \mathrm{~b}$ ) showed that the size of the imitative compatibility effect reduced across the course of a block of trials, whereas the spatial compatibility effect remained constant. They interpreted this interaction, between compatibility modality and stage within the block, as indicating the presence of different mechanisms for spatial compatibility and imitative compatibility. 
However, there are two problems with the above interpretation: first, the two effects were assessed using different tasks with different stimulus processing demands. Spatial compatibility was measured by asking participants to imitate the identity of the finger that was performing a tapping movement; this finger could be either spatially compatible or incompatible with the participant's movement. Imitative compatibility was determined by instructing participants to match spatially the finger that was performing a tapping movement; this finger could be either imitatively compatible or incompatible with the participant's movement. Thus, the spatial compatibility task required analysis of the finger identity, while the imitative compatibility task required analysis of the spatial location of the finger. It is likely that these tasks take a different amount of time to perform. Indeed, response times appear to have been longer for the spatial compatibility experiment, where participants had to process the finger identity, which is a more complex task than processing its spatial location. The current Experiments 1 and 2 suggest that the relative size of spatial compatibility and imitative compatibility effects may alter with increasing response time, which makes this a potentially problematic confound: it may be that in the more simple imitative compatibility task, response times were too short to allow a reliable imitative compatibility effect to emerge.

The second and more critical obstacle in interpreting the results of Bertenthal et al. (2006) is that compatible and incompatible trials were presented in separate blocks. This allows the development of response strategies as the block progresses. For example, in the spatial compatibility experiment (Experiment 3a), where the instruction was to imitate the identity of the moving finger, a valid strategy on a (spatially) compatible block would be instead to match the spatial location, which is an easier discrimination to make and therefore can be performed more quickly. Because the trials are blocked, this strategy could develop across a block, once the participant realises the spatially compatible nature of the trials. Indeed, the 
spatial compatibility effect in Experiment 3a showed a trend towards a linear increase across the four quarters of each block, driven by a decrease in response times on spatially compatible trials. In contrast, in the imitative compatibility experiment (Experiment 3b), the effect decreased across the four quarters of each block, driven by a decrease in response times on imitatively incompatible trials. The instruction here was to match the spatial location of the moving finger. It is possible that participants could develop a strategy to avoid interference during an imitatively incompatible block, which would allow them not to process the incompatible imitative attribute of the moving finger while preserving spatial information. Again, the blocked trials would allow this strategy to develop once the participant realises the imitatively incompatible nature of the block. Thus, alternative response strategies, driven by the differing task demands and the blocked presentation of compatible and incompatible trials, could explain the pattern of data observed by Bertenthal et al. (2006) without the need to invoke two distinct psychological mechanisms.

Since the current experiments used the same task to measure both spatial and imitative compatibility, and trial types were fully randomised, it is possible to contrast the results of Bertenthal et al. (2006) with the results of Experiment 1 which comprised four consecutive blocks of trials. If Bertenthal et al. (2006) are correct, and spatial compatibility and imitative compatibility are the result of different mechanisms which progress at different rates across the course of an experiment, then there should be an interaction between the size of the two effects across the four blocks of Experiment 1: the imitative compatibility effect should reduce, while the spatial compatibility effect should remain constant. The sizes of the imitative compatibility and spatial compatibility effects were therefore calculated for each block and entered into repeated measures ANOVA with within-subjects factors of block (1 4) and compatibility modality (spatial, imitative). There was a main effect of compatibility modality: as noted previously, the spatial compatibility effect was greater than the imitative 
compatibility effect $\left(F_{1,15}=39.4, p<0.001\right)$. There was no main effect of block and, contrary to the findings of Bertenthal et al. (2006), no interaction between block and compatibility modality $\left(F_{3,45}<1\right)$.

Independent Effects Arising from Common Mechanisms

It therefore appears that, when the same task is used to measure both spatial compatibility and imitative compatibility, and when trials are randomised such that alternative response strategies cannot be used, there is no evidence for differential progression of the two processes across trials within an experiment. While it is difficult to form firm conclusions on the basis of a null result, when task differences and alternative response strategies are eliminated there seems to be little evidence for the presence of different underlying mechanisms contributing to imitative and spatial compatibility. Although the present experiments demonstrate that spatial compatibility and imitative compatibility are independent of one another, there is as yet no evidence to contradict the suggestion that these processes arise, independently, from the same domain-general processes of associative learning and stimulus-response compatibility.

Implications for Research on Imitation

The present experiments support the validity of imitative compatibility as a measure of imitation. This result, therefore, supports the conclusions drawn from the studies discussed in the Introduction, which used imitative compatibility to investigate the mechanisms, functions, and developmental origins of imitation. Convergent evidence relating to the developmental origins of imitation may be obtained through comparison of the effects of sensorimotor training on imitative compatibility and on neurological measures of mirror system 
functioning. Incompatible sensorimotor training, where participants respond to the observation of an action with the performance of a non-matching action, abolishes imitative compatibility (Gillmeister et al., 2008; Heyes et al., 2005). It has recently been demonstrated that the same type of incompatible sensorimotor training reverses both muscle-specific motor cortical excitability (Catmur, Walsh, \& Heyes, 2007) and the dominance for hand over foot actions in mirror system areas (Catmur, Gillmeister, Bird, Liepelt, Brass \& Heyes, 2008) during passive action observation.

As discussed in the Introduction, imitation requires perceptual-motor translations between observed and executed movements: the kind of translations which are thought to underlie imitative compatibility. By demonstrating that imitative compatibility is independent of simple spatial compatibility, and that the two processes follow different time courses within trials, the current experiments support the use of imitative compatibility as a measure of imitation. 


\section{References}

Aicken, M. D., Wilson, A. D., Williams, J. H., \& Mon-Williams, M. (2007). Methodological issues in measures of imitative reaction times. Brain and Cognition, 63, 304-308.

Bertenthal, B. I., Longo, M. R., \& Kosobud, A. (2006). Imitative response tendencies following observation of intransitive actions. Journal of Experimental Psychology: Human Perception and Performance, 32, 210-225.

Bird, G., Leighton, J., Press, C., \& Heyes, C. (2007). Intact automatic imitation of human and robot actions in autism spectrum disorders. Proceedings of the Royal Society B: Biological Sciences, 274, 3027-3031.

Brass, M., Bekkering, H., \& Prinz, W. (2001). Movement observation affects movement execution in a simple response task. Acta Psychologica (Amsterdam), 106, 3-22.

Brass, M., Bekkering, H., Wohlschlager, A., \& Prinz, W. (2000). Compatibility between observed and executed finger movements: comparing symbolic, spatial, and imitative cues. Brain and Cognition, 44, 124-143.

Catmur, C., Gillmeister, H., Bird, G., Liepelt, R., Brass, M., \& Heyes, C. (2008). Through the looking glass: counter-mirror activation following incompatible sensorimotor learning. European Journal of Neuroscience, 28, 1208-1215.

Catmur, C., Walsh, V., \& Heyes, C. (2007). Sensorimotor learning configures the human mirror system. Current Biology, 17, 1527-1531.

Catmur, C., Walsh, V., \& Heyes, C. (2009). Associative sequence learning: the role of experience in the development of imitation and the mirror system. Philosophical Transactions of the Royal Society Series B, 364, 2369-2380. 
Chartrand, T. L. \& Bargh, J. A. (1999). The chameleon effect: the perception-behavior link and social interaction. Journal of Personality and Social Psychology, 76, 893-910.

Cho, Y. S. \& Proctor, R. W. (2004). Influences of multiple spatial stimulus and response codes on orthogonal stimulus-response compatibility. Perception and Psychophysics, 66, 1003-1017.

Craighero, L., Bello, A., Fadiga, L., \& Rizzolatti, G. (2002). Hand action preparation influences the responses to hand pictures. Neuropsychologia, 40, 492-502.

di Pellegrino, G., Fadiga, L., Fogassi, L., Gallese, V., \& Rizzolatti, G. (1992). Understanding motor events: a neurophysiological study. Experimental Brain Research, 91, 176-180.

Edwards, M.G., Humphreys, G.W., \& Castiello, U. (2003). Motor facilitation following action observation: a behavioural study in prehensile action. Brain and Cognition, 53, 495502.

Fadiga, L., Fogassi, L., Pavesi, G., \& Rizzolatti, G. (1995). Motor facilitation during action observation: a magnetic stimulation study. Journal of Neurophysiology, 73, 2608-2611.

Gallese, V., Fadiga, L., Fogassi, L., \& Rizzolatti, G. (1996). Action recognition in the premotor cortex. Brain, 119 ( Pt 2), 593-609.

Gillmeister, H., Catmur, C., Liepelt, R., Brass, M., \& Heyes, C. (2008). Experience-based priming of body parts: A study of action imitation. Brain Research, 1217, 157-170.

Hari, R., Forss, N., Avikainen, S., Kirveskari, E., Salenius, S., \& Rizzolatti, G. (1998). Activation of human primary motor cortex during action observation: a neuromagnetic study. Proceedings of the National Academy of Sciences of the United States of America, 95, 1506115065. 
Heiser, M., Iacoboni, M., Maeda, F., Marcus, J., \& Mazziotta, J. C. (2003). The essential role of Broca's area in imitation. European Journal of Neuroscience, 17, 1123-1128.

Heyes, C., Bird, G., Johnson, H., \& Haggard, P. (2005). Experience modulates automatic imitation. Brain Research Cognitive Brain Research, 22, 233-240.

Heyes, C. M. \& Ray, E. D. (2000). What is the significance of imitation in animals? Advances in the Study of Behavior, 29, 215-245.

Hommel, B. (1993). The role of attention for the Simon effect. Psychological Research, 55, 208-222.

Hommel, B. (1994). Spontaneous decay of response-code activation. Psychological Research, 56, 261-268.

Hommel, B., Musseler, J., Aschersleben, G., \& Prinz, W. (2001). The Theory of Event Coding (TEC): a framework for perception and action planning. Behavioral and Brain Sciences, 24, 849-878.

Iacoboni, M. \& Mazziotta, J. C. (2007). Mirror neuron system: basic findings and clinical applications. Annals of Neurology, 62, 213-218.

Iacoboni, M., Woods, R. P., Brass, M., Bekkering, H., Mazziotta, J. C., \& Rizzolatti, G. (1999). Cortical mechanisms of human imitation. Science, 286, 2526-2528.

Jansson, E., Wilson, A. D., Williams, J. H., \& Mon-Williams, M. (2007). Methodological problems undermine tests of the ideo-motor conjecture. Experimental Brain Research, 182, $549-558$. 
Kilner, J. M., Hamilton, A. F., \& Blakemore, S. J. (2007). Interference effect of observed human movement on action is due to velocity profile of biological motion. Social Neuroscience, 2, 158-166.

Kilner, J. M., Paulignan, Y., \& Blakemore, S. J. (2003). An interference effect of observed biological movement on action. Current Biology, 13, 522-525.

Kornblum, S., Hasbroucq, T., \& Osman, A. (1990). Dimensional overlap: cognitive basis for stimulus-response compatibility - a model and taxonomy. Psychological Review, 97, 253270.

Liepelt, R., von Cramon, D. Y., \& Brass, M. (2008). What is matched in direct matching? Intention attribution modulates motor priming. Journal of Experimental Psychology: Human Perception and Performance, 34, 578-591.

Longo, M. R. \& Bertenthal, B. I. (2009). Attention modulates the specificity of automatic imitation to human actors. Experimental Brain Research, 192, 739-744.

Longo, M. R., Kosobud, A., \& Bertenthal, B. I. (2008). Automatic imitation of biomechanically possible and impossible actions: Effects of priming movements versus goals. Journal of Experimental Psychology: Human Perception and Performance, 34, 489501.

Nicoletti, R. \& Umiltà, C. (1984). Right-left prevalence in spatial compatibility. Perception and Psychophysics, 35, 333-343.

Press, C., Bird, G., Flach, R., \& Heyes, C. (2005). Robotic movement elicits automatic imitation. Brain Research Cognitive Brain Research, 25, 632-640.

Press, C., Gillmeister, H., \& Heyes, C. (2006). Bottom-up, not top-down, modulation of imitation by human and robotic models. European Journal of Neuroscience, 24, 2415-2419. 
Press, C., Gillmeister, H., \& Heyes, C. (2007). Sensorimotor experience enhances automatic imitation of robotic action. Proceedings of the Royal Society B: Biological Sciences, 274, 2509-2514.

Ratcliff, R. (1979). Group reaction time distributions and an analysis of distribution statistics. Psychological Bulletin, 86, 446-461.

Ray, E. B. \& Heyes, C. M. (in press). Imitation in infancy: the wealth of the stimulus. Developmental Science.

Stürmer, B., Aschersleben, G., \& Prinz, W. (2000). Correspondence effects with manual gestures and postures: a study of imitation. Journal of Experimental Psychology: Human Perception and Performance, 26, 1746-1759.

Tagliabue, M., Zorzi, M., Umiltà, C., \& Bassignani, F. (2000). The role of long-termmemory and short-term-memory links in the Simon effect. Journal of Experimental Psychology: Human Perception and Performance, 26, 648-670.

Vainio, L., Tucker, M., \& Ellis, R. (2007). Precision and power grip priming by observed grasping. Brain and Cognition, 65, 195-207.

Vogt, S., Taylor, P., \& Hopkins, B. (2003). Visuomotor priming by pictures of hand postures: perspective matters. Neuropsychologia, 41, 941-951. 